BULL. AUSTRAL. MATH. SOC.

VOL. 32 (1985), 465-467.

\title{
ROTATING MAGNETIC UPPER-MAIN SEQUENCE STARS
}

\section{E. GALEA}

The subject of this thesis is the theoretical description of the magnetic fields observed in early type upper-main sequence stars of spectral classification $A p / B p$.

Observational evidence strongly supports the oblique rotator model in which the magnetic axis of symetry is inclined to the rotation axis of the star at what appears to be a random angle, $X$ (see chapter 1). Magnetic field geometries consisting of either a centred dipole plus contributions from higher order components or a decentred dipole seem best to model observations.

Within the framework of the oblique rotator model the steady state structure of uniformly rotating magnetic stars is analysed using first order perturbation theory (see Chapters 2 and 3). Such an analysis involves the solution of a system of highly non-linear first order differential equations. The solution is achieved by the use of a NewtonRaphson iteration procedure (see Chapter 4).

Equilibrium models in which radiative equilibrium is strictly adhered to are first examined (see Chapters 3, 5 and 6). A number of magnetic geometries are considered. Two basic model types are examined. In the first we express the magnetic field in terms of quantities which are symmetric with respect to an axis which is inclined to the rotation axis.

Received 15 June 1985. Thesis submitted to University of Newcastle, November 1984. Degree approved May 1985. Supervisor: Dr. W. P. Wood.

Copyright Clearance Centre, Inc. Serial-fee code: 0004-9727/85 $\$ A 2.00+0.00$. 
These models involve the parameter $X$. Models possessing poloidal only and both poloidal and toroidal magnetic field components (with respect to the magnetic axis) are constructed (see Chapter 3). For $x$ less than or approximately equal to fifty-five degrees the results are analogous to the results found for aligned models ( $x$ equals zero degrees) which have been reported previously in the literature. For $X$ greater than or approximately equal to fifty-five degrees the models exhibit the behaviour of the perpendicular models ( $x$ equals ninety degrees). The oblique models with $\chi$ greater than or approximately equal to fifty-five degrees share with the perpendicular models the lack of convergence for rotation speeds and field strengths relevant to the magnetic stars (see Chapter 5). The dichotomy in trends between the aligned and perpendicular models is shown to be a direct result of the difference in the geometries assumed for the magnetic field.

The second type of magnetic geometry considered involves certain levels of truncation in the infinite series representation of a general magnetic field which is a function of three co-ordinate axes. The system of perturbation equations which results from this type of analysis is overdetermined and different choices for the determined system lead to quantitatively different results. By insisting that all components of the perturbed hydrostatic equations balance, the arbitrariness in the choice of systems of equations is removed (see Chapter 3). Usinq this representation of the magnetic field, linear relationships occur between the various radial components of the magnetic field expansion. For the simple models of Chapter 3 , it is possible to relate the arbitrary constant of proportionality to the obliquity angle $x$. For more complicated field expansions involving additional terms in the magnetic expansion, more arbitrary constants appear and their physical significance is not clear (see Chapter 6). From this analysis it appears that certain levels of truncation of the infinite vector spherical harmonic series are equivalent to truncated series representations for an axi-symetric oblique field.

In addition to the above models, models possessing oblique magnetic geometries in which the strict radiative equilibrium constraint is relaxed are also constructed. This results in a slow meridional flow in the radiative region of the star. The inclusion of a meridional 
circulation current merely enhances the geometrically based differences found in the 'aligned' type and 'perpendicular' type rotators (see Chapter 7).

University of St. Andrews,

Mathematical Institute,

Department of Applied Mathematics,

Fife KY16 9SS,

United Kingdom. 